\title{
Canadian Society of Transplantation consensus guidelines on eligibility for kidney transplantation
}

\author{
Greg Knoll, Sandra Cockfield, Tom Blydt-Hansen, Dana Baran, Bryce Kiberd, David Landsberg, \\ David Rush, Edward Cole, for the Kidney Transplant Working Group of the Canadian Society of \\ Transplantation
}

The full-text version of the Canadian Society of Transplantation consensus guidelines on eligibility for kidney transplantation is available at www.cmaj.ca/cgi/content/full/I73/Io/II8I/DCI.

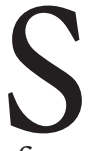

ince 1980 the incidence and prevalence of end-stage renal disease have grown each year in Canada and throughout the world. From I98I to 1999, the number of new patients with the disease increased at a compound annual rate of $7 \cdot 3 \%,{ }^{1}$ and similar trends have been documented worldwide. By the end of 2000, 24 92I Canadians were receiving life-sustaining treatment for end-stage renal disease, of whom I4 567 were receiving dialysis and Io 354 had a functioning kidney transplant. ${ }^{2}$ End-stage renal disease is associated with premature death and a substantial reduction in health-related quality of life. Kidney transplantation is the treatment of choice because it prolongs survival, improves quality of life and is less costly than dialysis.

Despite the benefits of kidney transplantation, not all patients with end-stage renal disease proceed with transplantation, and there is considerable variation in transplantation rates across Canada. ${ }^{2}$ For example, the renal transplantation rate (per million population) is only 27.4 in Saskatchewan compared with $5 \mathrm{I} .8$ in the Atlantic provinces. ${ }^{2}$ It is not known how much of this variation is due to differences in referral and acceptance for transplantation (i.e., perceived eligibility) or differences in the availability of donors.

In an effort to outline which patients in the growing population with end-stage renal disease are currently suitable for transplantation, the Canadian Society of Transplantation formed a committee to develop consensus guidelines on eligibility criteria. Committee members were selected on the basis of geographical representation as well as specialty (adult and pediatric nephrology). Nineteen topics important in determining eligibility for transplantation were chosen for inclusion in the guidelines. After a detailed review of the literature, a draft document was created by the committee and circulated to transplant programs across the country for comments and input. The draft guidelines were reviewed and revised at the Canadian Society of Transplantation Annual Meeting. A consultative approach was used to ensure that the recommendations reflected a Canadian consensus and would be adopted across the country. The full text of the recommendations is available online at www.cmaj.ca/cgi/content/full/I73/Io /II8I/DCI.

Of all the topics reviewed, perhaps the most difficult was that of advancing age. Over the past decade, the proportion of dialysis patients over the age of 65 has increased markedly. In fact, by 2003 , almost $54 \%$ of patients starting renal replacement therapy were in this age group. ${ }^{3}$ On the other hand, improved patient and graft survival with current immunosuppressive protocols has made kidney transplantation possible for selected patients over age 65. Although life expectancy is less, such recipients have death-censored graft survival rates that are at least as good as those of younger patients. The guidelines recommend that advanced age alone not be a contraindication to transplantation. Transplant candidates should have a reasonable probability of surviving beyond current waiting times for transplantation, given the resources required to assess and maintain patients on a waiting list. The decision regarding eligibility for transplantation must be made in the best interests of the patient and on medical and surgical grounds.

These guidelines were developed with a wide audience in mind. General recommendations are given in summary form for review by health care workers and physicians working in primary care who may want to know whether their patient is eligible for transplantation. Further detail expanding on the recommendations is also provided. The guidelines refer to both adult and pediatric patient groups.

We hope that these guidelines will lead to consistency in determining which patients are eligible and accepted for kidney transplantation. They are based on the best available evidence, with the understanding that clinical judgement plays a role in decision-making. As such, there will likely still be variability in clinical practice across the country. This consensus document specifically addresses eligibility criteria for kidney transplantation and does not outline the individual tests required for assessing or reassessing patients awaiting kidney transplantation, since guidelines from the United States and Europe already provide this information. ${ }^{4-6}$ 
This is an abridged summary of the guidelines and levels of evidence supporting the reccommendations. We used the grading scheme developed by the Canadian Task Force on the Periodic Health Examination, ${ }^{7}$ where $A$ is the highest level of evidence supporting the recommendation, $B$ represents fair evidence in support of the recommendation, $C$ represents conflicting evidence melded with expert opinion, and $D$ and $E$ represent evidence against a recommendation. In our review grades $\mathrm{D}$ and $\mathrm{E}$ were never applicable and can be omitted. These recommendations have been edited for brevity, and the reader should, for specific cases and conditions, consult the complete document online.

\section{Executive summary of recommendations for transplantation}

\section{General considerations}

I. All patients with end-stage renal disease (ESRD) should be considered for kidney transplantation. (Grade A)

2. Eligibility for kidney transplantation should be determined on medical and surgical grounds and not on social status, sex, ethnicity or personal or public appeal. (Grade C)

3. A patient declined for transplantation should routinely be offered a second opinion. (Grade C)

\section{Timing of referral}

I. Potential transplant recipients should be referred for evaluation by a transplant program once renal replacement therapy is expected to be required within the next 12 months. (Grade C)

2. Patients already requiring dialysis support should be referred for transplant evaluation as soon as their medical condition stabilizes. (Grade C)

\section{Renal function}

I. Preemptive kidney transplantation is the preferred form of renal replacement therapy. (Grade A)

2. Preemptive kidney transplantation should not proceed unless the measured or calculated glomerular filtration rate is less than $20 \mathrm{~mL} / \mathrm{min}$ and there is evidence of progressive and irreversible deterioration in renal function over the previous 6-I2 months. (Grade C)

\section{Age and functional capacity}

I. Advanced age per se is not a contraindication to kidney transplantation. (Grade B)

2. Transplant candidates should have a reasonable probability of surviving beyond current waiting times for transplantation, given the resources required to assess and maintain patients on the renal transplant waiting list. (Grade C)

3. Very young age and small size should not prevent early referral for transplant evaluation. (Grade B)

4. Cognitive or neurodevelopmental delay is not an absolute contraindication to renal transplantation in children. (Grade B)

\section{Obesity}

I. Few data exist to suggest which, if any, obese (body mass index $30 \mathrm{~kg} / \mathrm{m}^{2}$ ) patients should be denied transplantation on the basis of obesity per se. (Grade C)

\section{Cause of end-stage renal disease}

I. There are few contraindications to kidney transplantation solely on the basis of the cause of ESRD. (Grade A)

\section{Systemic diseases}

I. Systemic diseases leading to ESRD are usually not a contraindication to kidney transplantation; however, the presence and severity of extra-renal disease will often determine whether transplantation is an option. (Grade C)

2. Patients with the following conditions should be considered for kidney transplantation:

- Diabetes mellitus (Grade B). Simultaneous kidneypancreas transplantation should be considered in selected patients with type I diabetes mellitus.

- Primary hyperoxaluria (Grade B)

- Fabry disease (Grade B)

- Sickle-cell disease (Grade B)

- Anti-glomerular basement membrane disease (Grade C)

- Amyloidosis (primary or secondary) (Grade B)

- Systemic lupus erythematosus (Grade C)

- Scleroderma (Grade C)

- Vasculitis (Wegener's granulomatosis, microscopic polyangiitis, pauci-immune necrotizing glomerulonephritis, Henoch-Schonlein purpura) (Grade C)

- Thrombotic microangiopathy or hemolytic uremic syndrome (Grade C)

- Congenital nephrotic syndrome (Grade B)

- Cystinosis (Grade B)

- Autosomal recessive polycystic kidney disease (Grade A)

\section{Infection}

I. Patients should be free of active infection, whether of viral, bacterial or fungal origin. (Grade B)

2. Where possible, transplant candidates should be vaccinated against infections that are prevalent or potentially life-threatening. (Grade A)

3. Transplant candidates should be screened for exposure to mycobacteria with a careful clinical history, chest radiography and purified protein derivative skin testing. (Grade C)

4. Serostatus for cytomegalovirus and Epstein-Barr virus should be assessed before transplant but should not determine eligibility for transplantation. (Grade A)

5. All patients being assessed for kidney transplantation should be screened for HIV infection (Grade A). HIVinfected patients with end-stage kidney failure may be considered for kidney transplantation if they demonstrate adherence to antiretroviral therapy and have stable disease. (Grade B) 


\section{Malignant disease}

I. Renal transplant candidates with a previous history of malignant disease should be tumour-free before proceeding with transplantation (Grade A). Renal transplant candidates with a history of malignant disease should wait a period of time between successful treatment and transplantation. The length of time will depend on the type of malignancy (Grade B). The guidelines have recommendations for most of the common cancers.

\section{Pulmonary disease}

I. Patients with severe respiratory conditions are not candidates for kidney transplantation (Grade C). The guidelines have detailed recommendations regarding severity of respiratory disease, specific respiratory diseases such as asthma and chronic obstructive pulmonary disease and for respiratory diseases in children.

\section{Cardiac disease}

I. All patients should be assessed for the presence of ischemic heart disease and left ventricular dysfunction before kidney transplantation (Grade A). The guidelines have recommendations for transplantation eligibility based on different cardiac conditions and the results of screening tests (if appropriate).

\section{Cerebral vascular disease}

I. Kidney transplantation should be deferred in patients with a history of stroke or transient ischemic attack for at least 6 months following the event. (Grade A)

\section{Peripheral vascular disease}

I. The presence of pretransplant peripheral vascular disease is not an absolute contraindication to kidney transplantation. However, the risk of death is increased, and the presence of peripheral vascular disease should be considered in the context of other comorbidities in determining eligibility for kidney transplantation. (Grade B)

2. Patients with large uncorrectable abdominal aneurysms, severe occlusive common iliac disease, active gangrene or recent atheroembolic events are not candidates for kidney transplantation. (Grade C)

\section{Gastrointestinal disease}

I. Patients with active peptic ulcer disease should not receive a transplant until the disease is successfully treated. (Grade C)

2. The presence of asymptomatic cholelithiasis is not a contraindication to kidney transplantation. (Grade A)

3. Patients with previous cholecystitis or suggestive symptoms should be investigated for the presence of gallstones. If gallstones are found, these patients should be considered for cholecystectomy before kidney transplantation. (Grade C)
4. There are specific recommendations for patients with diverticulitis, acute and chronic pancreatitis and inflammatory bowel disease in the guidelines.

\section{Liver disease}

I. All transplant candidates should be screened for evidence of liver disease including hepatitis B and C virus. (Grade C)

2. Patients who are hepatitis B surface antigen positive or hepatitis $\mathrm{C}$ antibody positive should be considered for transplantation. However, eligibility will depend on other considerations such as viral load and liver function and liver histology. (Grade C)

3. Transplant candidates with cirrhosis should not be considered for kidney transplantation alone, but may be considered for combined liver-kidney transplantation. (Grade C)

\section{Genitourinary disease}

I. A urologic cause of ESRD is not necessarily a contraindication to kidney transplantation provided appropriate urinary tract drainage can be achieved. (Grade A)

\section{Hematologic disorders}

I. The presence of thrombophilia, hypercoagulable state or cytopenia is not an absolute contraindication to kidney transplantation, but these conditions should be fully investigated. (Grade C)

2. Patients requiring long-term anticoagulation for recurrent deep venous thrombosis, atrial fibrillation, prosthetic heart valves or hypercoagulable states are candidates for kidney transplantation. (Grade C)

\section{Hyperparathyroidism}

I. Calcium, phosphorus and parathyroid hormone levels should be measured as part of the pretransplant evaluation (Grade A), and parathyroidectomy should be considered for those in whom medical management has not worked or those with severe, persistent complications of hyperparathyroidism.

\section{Psychosocial considerations}

I. All patients should have a pretransplant psychosocial evaluation by an experienced competent individual to assess for cognitive impairment, mental illness, nonadherence to therapy and drug or alcohol abuse. (Grade C)

2. Patient nonadherence to therapy is a contraindication to kidney transplantation (Grade A). Kidney transplantation should be delayed until patients have demonstrated adherence to therapy for at least 6 months (Grade C).

3. Kidney transplantation should be delayed until the patient has demonstrated freedom from substance abuse for at least 6 months. (Grade C)

4. Cognitive impairment is not an absolute contraindication to kidney transplantation. (Grade B) 
Greg Knoll is at the Division of Nephrology, University of Ottawa and The Ottawa Hospital, Ottawa, Ont. Sandra Cockfield is at the Division of Nephrology, University of Alberta Hospital, Edmonton, Alta. Tom BlydtHansen is at the Division of Pediatric Nephrology, University of Manitoba, Winnipeg, Man. Dana Baran is at the Division of Nephrology, McGill University Health Centre, Montréal, Qué. Bryce Kiberd is at the Division of Nephrology, Queen Elizabeth II Health Sciences Centre, Halifax, NS. David Landsberg is at the Division of Nephrology, St. Paul's Hospital, University of British Columbia, Vancouver, BC. David Rush is at the Division of Nephrology, University of Manitoba, Winnipeg, Man. Edward Cole is at the Division of Nephrology, University of Toronto, Toronto, Ont.

Competing interests: None declared.

Contributors: All of the authors contributed equally to the writing and revision of the manuscript and approved the final version submitted for publication.

\section{REFERENCES}

Acknowledgements: The Kidney Transplant Working Group received unrestricted grants from the following organizations to hold the in-person meetings needed to create the guidelines: Fujisawa Canada (now known as Astellas Pharma Canada), Novartis Pharmaceuticals Canada, Hoffmann-La Roche, Wyeth Canada and SangStat Canada (now known as Genzyme Canada). In addition, the Kidney Transplant Working Group thank the Canadian Council for Donation and Transplantation, who provided financial support to publish the guidelines.
I. Canadian Institute for Health Information. Canadian Organ Replacement Register. 200I Report, Volume I: dialysis and renal transplantation. Ottawa: Aug. I, 200I.

2. Canadian Institute for Health Information. Canadian Organ Replacement Register. Preliminary report for dialysis and transplantation 2002. Ottawa: 2002. Available: http://secure.cihi.ca/cihiweb/dispPage.jsp?cw_page=reports_corr2002prelim_e (accessed 2005 Oct 4 ).

3. Canadian Institute for Health Information. Canadian Organ Replacement Regis ter. Older end-stage renal disease patients on renal replacement therapy in Canada; incidence, prevalence, and treatment patterns. CORR inSITES, June 2005 Available: http://secure.cihi.ca/cihiweb/dispPage.jsp?cw_page=reports_corrinsites_jun2005_e (accessed 2005 Oct 4).

4. Kasiske BL, Cangro CB, Hariharan S, et al., for the American Society of Transplantation.The evaluation of renal transplant candidates: clinical practice guidelines. Am J Transplant 2001;2(Suppl I):I-95.

5. European Best Practice Guidelines Expert Group on Renal Transplantation. Section I: Evaluation, selection and preparation of the potential transplant recipient. Nephrol Dial Transplant 2000;15(Suppl 7):3-38.

6. Danovitch GM, Hariharan S, Pirsch JD, et al. Management of the waiting list for cadaveric kidney transplants: report of a survey and recommendations by the Clinical Practice Guidelines Committee of the American Society of Transplantation. Am Soc Nephrol 2002;13:528-35.

7. Canadian Task Force on the Periodic Health Examination. Task Force Report on the Periodic Health Examination. CMAJ I979;I2I:II93-I254.

Correspondence to: Dr. Greg Knoll, Division of Nephrology, The Ottawa Hospital, Riverside Campus, 1967 Riverside Dr., Ottawa ON KIH 7 W9; gknoll@ottawahospital.on.ca

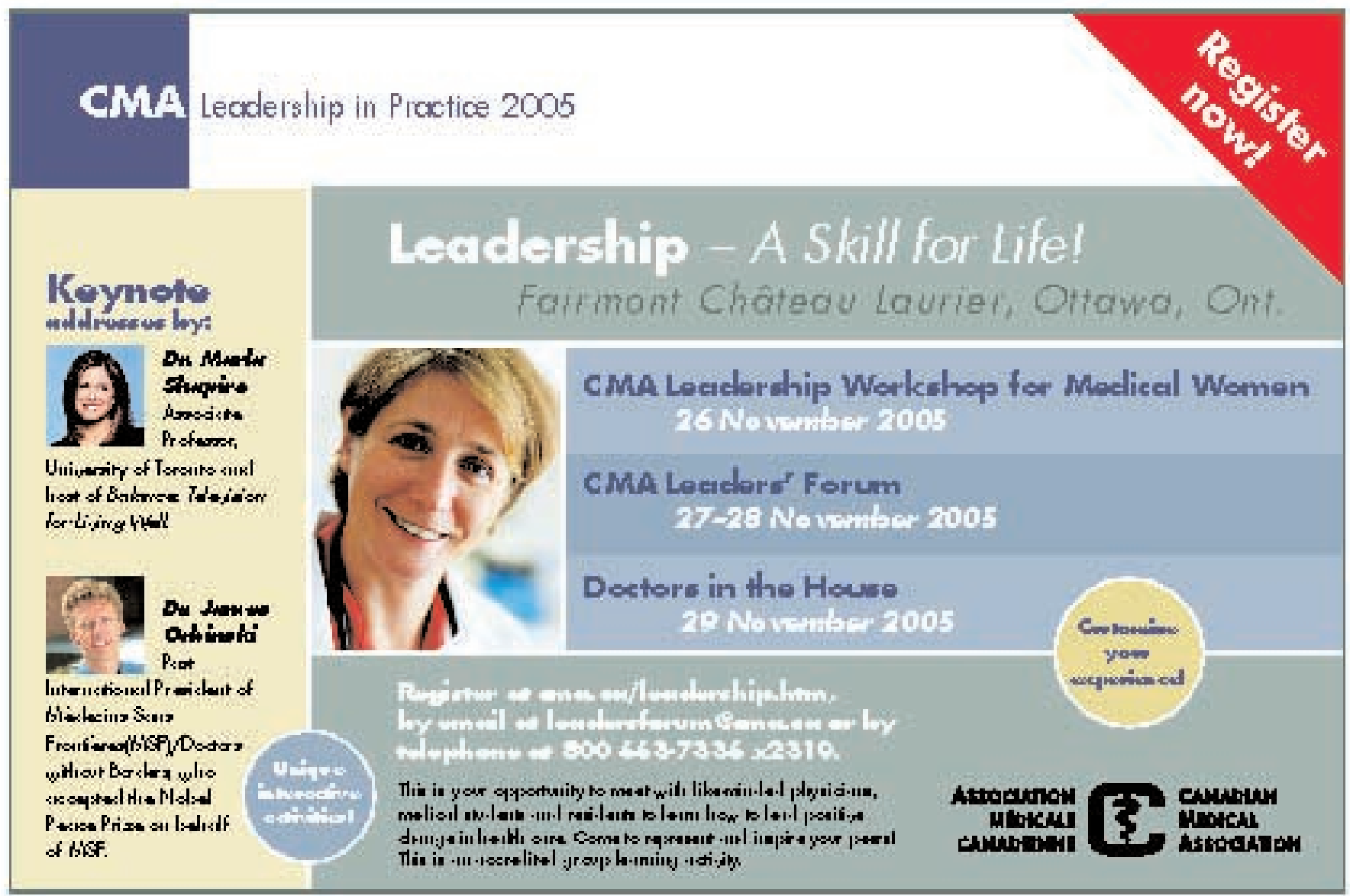

